2019-05-27

\title{
Platooning Control for Sailboats Using a Tack Strategy
}

viel, christophe

http://hdl.handle.net/10026.1/14221

10.1007/s12555-018-0291-7

International Journal of Control, Automation and Systems

Springer Verlag

All content in PEARL is protected by copyright law. Author manuscripts are made available in accordance with publisher policies. Please cite only the published version using the details provided on the item record or document. In the absence of an open licence (e.g. Creative Commons), permissions for further reuse of content should be sought from the publisher or author. 


\title{
Platooning control for sailboats using a tack strategy
}

\section{Christophe VIEL, Ulysse VAUTIER, Jian WAN, and Luc JAULIN}

\begin{abstract}
This paper addresses the problem of platooning control for a fleet of sailboats. A quadrilateral path is proposed to avoid going into the wind. A tack strategy is defined to go front the wind, stay in a short corridor and regulate the sailboat acceleration, which is a challenging task in comparison to autonomous underwater vehicles (AUVs) or surface vehicles that can use motors for such regulation. Desired acceleration which guarantees to reach the platooning has been derived and validated. A method of regulating the sail angle using a proportional command is proposed to control the sailboat acceleration. Simulation results demonstrate the effectiveness of the proposed approach.
\end{abstract}

Keywords: Autonomous sailboat, non-linear control, platooning.

\section{INTRODUCTION}

For the past decades, the needs of oceanographic measurement and observation have stimulated marine robotics research. Considerable progresses have been made in the development and the use of autonomous marine platforms for ocean exploration.

Despite of their limited speed and wind dependence, sailboats have a high potential for research measurement because they rely on renewable solar and wind energies for long-term missions. Other potential applications like surveillance and mapping create a growing interest for them. Autonomous robotic sailing is however faced with two inherent difficulties: the uncontrollable and partially unpredictable nature of wind direction and speed, and the complex kinematics and dynamics of a sailboat. This paper focuses on the platooning control for a fleet of sailboats rather than the control of an individual sailboat or the control of a fleet of other vehicles such cars or drones. Many low-level and high-level control systems designed for sailboats can be found in the literature [1-10] to follow line, avoid obstacles, path tracing or reach a target position.

Studying the platooning problem for sailboats is interesting in terms of synchronizing boats to take measurements such as combing an area to collect seafloor data and to find ship wreckages. It also allows to reach a common target, to avoid collisions while staying close to a position, to control the distance among sailboats when they return to harbors through a channel, etc. First created for avoiding traffic-jams, the goal of platooning is to maintain a desired distance among vehicles. A time headway is sometimes used to manage the desired distance among vehicles in the function of their velocities like in [11-13].
Since the topic of platooning has been mostly studied during the last decade for ground vehicles, it focuses more on specific topics like optimal control $[14,15]$, necessary sensor and communication schemes $[16,17]$, or reduction of communication using event-triggered methods [18-20]. Problems for surface and underwater vehicles have been studied in [21,22]. In these work, a distributed RHC (Receding Horizon Control) problem for nonlinear vehicle platooning with input and state constraints is developed, where an optimization allows keeping a stable platoon formation. Since in real-world applications, some information can be difficult to obtain due to disturbances like wind, wave and current conditions, neurodynamics observers are proposed in $[23,24]$ to recover the unmeasured velocity information and unknown vessel dynamics. Similarly, neurodynamic optimization and fussy approximation are used in [25] to evaluate unknown dynamics parameters. Control law of platooning is constructed based on the estimated parameters and an optimal guidance signal, shared by all vessels and obtained by the neurodynamic optimization.

For sailboats, platooning is more challenging to implement due to physical constraints like wind orientation, uncontrollability of sailboat velocity in some cases (upwind or backwind), and an absence of accurate control for the boat velocity in other cases. While AUVs and classic surface vehicles can control their position/velocity by using motors, exact sailboat position/velocity is uncontrollable, making previous strategies impossible to implement or follow straightforwardly. Thus, the main contributions of this paper are as follows:

- a control of sailboat orientation to regulate the sail- 
boat acceleration. This one allows performing a proposed platooning strategy, adapted for sailboat's specific problems, different from other marine vehicles. The proposed approach is compared with classic methods for showing its advantages.

- a simple method independent of sailboat dynamics and applicable to all kinds of sailboats. Only few sailboat parameters are required to implement it. To our knowledge, no paper treats the problem of platooning for sailboats so far.

Since a circle is difficult to follow in closed-loop platooning for a sailboat, a diamond trajectory is proposed, allowing to avoid the dead area of the wind and obtain a smoother control. Linear paths can also be considered. An evaluation of the desired acceleration to obtain a platooning has been defined and its convergence to the platooning has been proven. A tack strategy has been created to regulate the sailboat acceleration and to go front the wind. Performance obtained and problems of practical implementation for these two methods are compared. Finally, an illustrative example, consisting of the control of a sailboat fleet, is employed in order to demonstrate the proposed approach.

The outline of the paper is as follows. Problem statement and model equation of the sailboat are introduced in Section 2. Problem of platooning is described in Section 3: an approach to evaluate target acceleration and orientation of the sailboat to reach the platooning is proposed in Section 3.1 and 3.2, respectively. Low level control is presented in Section 4, with a method to choose rudder angle is defined Section 4.1 and a method to select sail angle in Section 4.2. Section 5 presents some simulation results and compares the proposed method with one in the literature. Section 6 concludes the paper.

\section{PROBLEM DESCRIPTION}

Consider a Multi-Agent System (MAS) consisting of a network of $N$ sailboats whose topology is described by a connected graph $\mathcal{G}=(\mathcal{N}, \mathcal{E})$, directed or undirected. $\mathcal{N}=\{1,2, \ldots, N\}$ is the set of nodes and $\mathcal{E} \subset \mathcal{N} \times \mathcal{N}$ the set of edges of the network. The sailboat $i$ is noted $S_{i}$. The set of neighbors of $S_{i}$ is $\mathcal{N}_{i}=\{j \in \mathcal{N} \mid(i, j) \in \mathcal{E}, i \neq j\}$. Let define the sailboat in front of $S_{i}$ is the $S_{i-1}$, where $S_{N}$ is the sailboat in front of $S_{1}$ if a platooning with closedloop path is considered, $S_{1}$ following a virtual leader with the fixed velocity $\bar{v}_{0}$ Let $1_{N}=\left[\begin{array}{llll}1 & 1 & \ldots & 1\end{array}\right]^{T} \in \mathbb{R}^{N}$ be the all-one vector and $I_{N} \in \mathbb{R}^{N \times N}$ be the identity matrix of size $N$. Let $L$ be the Laplacian matrix associated to $\mathcal{G}$. Remind $L$ is semi-definite positive, $L 1_{N}=0_{N}$ and $L$ is symmetric iff $\mathcal{G}$ is undirected.

Communications between sailboats are managed by the graph $\mathcal{G}$ which contains at least a spanning tree. Since the dynamics of the sailboat is much slower than the commu- nication time, consider the communication delay is negligible. When an sailboat $S_{i}$ broadcasts a message, it transmits the Cartesian position of its inertial center $P_{i}=\left[x_{i}, y_{i}\right]$, its velocity $v_{i}$ and its orientation $\theta_{i}$.

The following notation will be used in this paper:

- $\theta_{i}$ : orientation of the sailboat,

- $v_{i}$ : velocity of the sailboat,

- $\omega_{i}$ : rotation speed,

- $\phi_{i}$ : course angle,

- $\delta_{r, i}$ : angle of rudder, $\delta_{r, i} \leq \delta_{r, \max }$ taken as $\delta_{r, \max }=\frac{\pi}{4}$.

- $\delta_{s, i}$ : angle of sail, $\left|\delta_{s, i}\right| \leq \delta_{s, \max }$ taken as $\delta_{s, \max }=\frac{\pi}{2}$

- $\psi_{t w}, a_{t w}$ : orientation and speed of the true wind,

- $\delta$ : hauled angle, defining the dead area $[\psi+\pi-\delta, \psi+\pi+\delta] . \delta$ is taken equal to $\frac{\pi}{4}$.

- $M_{i}$ : sailboat $i$ mass.

- $L_{i}$ : length between the rudder and the keel.

- $l_{i}$ : the length between the keel and the bow.

Suppose $P_{i}, \theta_{i}, v_{i}, \dot{v}_{i}, \delta_{s, i}$ and $\delta_{r, i}$ can be measured by $S_{i}$. Moreover, suppose $\delta_{s, i}$ and $\delta_{r, i}$ are controllable.

The dynamics of the sailboat can be expressed with the general form

$$
\dot{x}_{i}=f_{i}\left(x_{i}, \delta_{r, i}, \delta_{s, i}\right)
$$

Note $f_{i}$ is always nonholonomic for a sailboat. Since a perfect knowledge of the dynamic parameters of the sailboat is difficult to obtain in practice, the proposed algorithm and control scheme in this paper are independent of the system $f_{i}\left(x_{i}, \boldsymbol{\delta}_{r, i}, \boldsymbol{\delta}_{s, i}\right)$ and use only parameters simple to measure such as $M_{i}, L_{i}$ and $l_{i}$. Thus, they can be adapted to various models of sailboats.

\subsection{True and apparent wind}

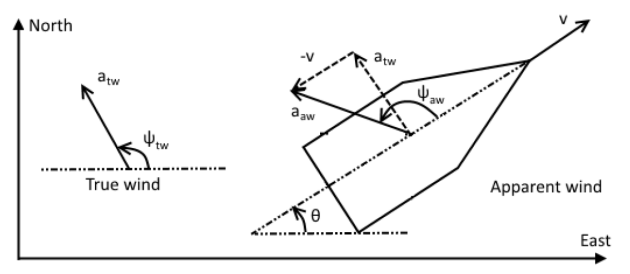

Fig. 1. [5] True wind, relative to an earth fixed point, and apparent wind, relative to the boat.

Wind can be described in two referential systems as true wind (tw) and apparent wind (aw), respectively. True wind, expressed in polar coordinate $W_{p, t w}$, is the velocity $a_{t w}$ and the direction of the air $\psi_{t w}$ measured from a platform fixed to the ground, in a fixed global coordinate. Apparent wind is the velocity and direction of the wind measured from a moving object, the wind felt on the ship in our case. Figure 1 illustrates apparent and true wind. 
Apparent wind relative to the direction of the boat can be evaluated from true wind in Cartesian coordinate by

$$
W_{c, a w}=\left[\begin{array}{c}
a_{t w} \cos \left(\psi_{t w}-\theta_{i}\right)-v \\
a_{t w} \sin \left(\psi_{t w}-\theta_{i}\right)
\end{array}\right]
$$

and in corresponding polar coordinate as

$$
W_{p, a w}=\left[\begin{array}{l}
a_{a w} \\
\psi_{a w}
\end{array}\right]=\left[\begin{array}{c}
\left|W_{c, a w}\right| \\
\operatorname{atan} 2\left(W_{c, a w}\right)
\end{array}\right]
$$

where $\operatorname{atan} 2$ is the arctangent function with two arguments returning an angle in the correct quadrant.

\section{PLATOONING CONTROL}

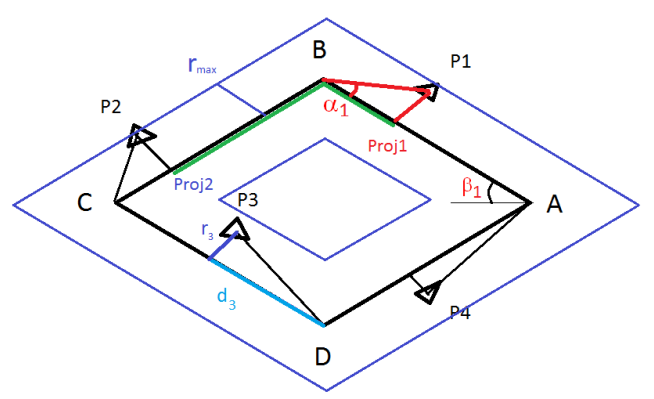

Fig. 2. Platooning parameters

Platooning problems studied in latest years focus on accurate methods to reduce the tracking error among vehicles. However, due to the unpredictable nature of the wind, wave and current conditions, the control of sailboat position/velocity is difficult or impossible in some orientations. Thus these strategies are inapplicable and the choice of a simple method adaptable to all kinds of sailboats has been made. The major contribution of this paper is the choice of the sailboat orientation exposed in Section 3.1 allowing to obtain a control of the sailboat acceleration to perform platooning strategies. A simple platooning control is exposed in Section 3.2.

In a classic closed-loop platooning for motor vehicles, turning around a circle is possible because the vehicle's velocity is independent of their orientation. For sailboats, wind orientation creates a dead area where boats can not sail directly. Thus, a quadrilateral ABCD of edges A, B, $\mathrm{C}$ and D (name its objectives) is defined as the path for our platooning so as to avoid this dead area. Sailboats follow edges in the order A, B, C and D, before back to A if a closed-loop is defined. More objectives can be used in the case where platooning with a non-closed path is considered. We also desire sailboats stay inside a corridor of size $2 r_{\text {max }}$ around each paths $\mathrm{AB}, \mathrm{BC}, \mathrm{CD}$ and $\mathrm{DA}$ and respect a distance $d_{\text {secu }}$ between them.

The following notations are defined and illustrated in the Figure 2:
- $\overline{\mathrm{O}}_{i}$ is the sailboat $i$ objective.

- $\underline{\mathrm{O}}_{i}$ is the sailboat $i$ previous objective. For example, if $\overline{\mathrm{O}}_{i}=\mathrm{C}$, one has $\underline{\mathrm{O}}_{i}=\mathrm{B}$.

- $\beta_{i}=\operatorname{angle}\left(\overline{\mathrm{O}}_{i} \underline{\mathrm{O}}_{i}\right)$ is the direction line.

- $d_{i}=\left\|\overline{\mathrm{O}}_{i} P_{i}\right\| \cos \left(\alpha_{i}\right)$ is the projection of the distance $\left\|\overline{\mathrm{O}}_{i} P_{i}\right\|$ on the axis $\overline{\mathrm{O}}_{i} \underline{\mathrm{O}}_{i}$, where $\alpha_{i}=\operatorname{angle}\left(\overline{\mathrm{O}}_{i} P_{i}\right)-\beta_{i}$

- $d_{i, i-1}$ is the projection of the distance between $S_{i}$ and $S_{i-1}$ on the path ABCD. Example: if $S_{i} \in \mathrm{AB}$ and $S_{i-1} \in \mathrm{CD}, d_{i, i-1}=\left|d_{i}+\|\mathrm{BC}\|+\left(\|\mathrm{CD}\|-d_{i-1}\right)\right|$

- $r_{i}=\left\|\overline{\mathrm{O}}_{i} P_{i}\right\| \sin \left(\alpha_{i}\right) \mid$ is the perpendicular distance between the sailboat and the axis $\overline{\mathrm{O}}_{i} \underline{\mathrm{O}}_{i}$.

- $v_{i}^{\text {proj }}=v_{i} \cos \left(\theta_{i}-\beta_{i}\right)$ and $\dot{v}_{i}^{\text {proj }}=\dot{v}_{i} \cos \left(\theta_{i}-\beta_{i}\right)$ are the projection of the velocity and acceleration of sailboat $i$ on the axis $\overline{\mathrm{O}}_{i} \underline{\mathrm{O}}_{i}$.

- $\bar{v}_{i}^{\text {proj }}$ is the desired acceleration of sailboat $i$, exposed in Section 3.2.

- $q_{i} \in\{-1,1\}$ is the tack variable

At $t=0$, the closer edge A, B, C, D to $S_{i}$ is chosen as the objective with its associated path, namely $\mathrm{AB}, \mathrm{BC}, \mathrm{CD}$ and DA. When distance $d_{i}$ between $S_{i}$ and its objective becomes smaller than a chosen value $d_{o b j}$, i.e. $d_{i}<d_{o b j}$, the next objective is chosen as the new objective of $S_{i}$. For example: if $\overline{\mathrm{O}}_{i}=\mathrm{A}$ and $d_{i}<d_{o b j}$ at the instant $t$, one has $\overline{\mathrm{O}}_{i}=\mathrm{B}$ and $\underline{\mathrm{O}}_{i}=\mathrm{A}$.

Remark 1: To obtain a platooning with smooth sailboat velocity, it is recommend to select $A B C D$ such as angles $\widehat{\mathrm{AB}}, \widehat{\mathrm{BC}}, \widehat{\mathrm{CD}}$ and $\widehat{\mathrm{DA}}$ are not inside the dead area of the wind, i.e. $\forall \beta \in\{\widehat{\mathrm{AB}}, \widehat{\mathrm{BC}}, \widehat{\mathrm{CD}}, \widehat{\mathrm{DA}}\}, \cos \left(\psi_{t w}-\beta\right)+$ $\cos (\delta)>0$.

\section{Constraints on $d_{\text {secu }}$}

Due to the weight, the size and the number of sailboats, some constraints on $d_{\text {secu }}$ must be respected to guarantee a feasible platooning. Indeed, to decelerate from sailboat maximal velocity $v_{\max }$ to 0 , a stopping distance must be maintained between the sailboat and the one in front of it. This condition can be expressed as

$$
d_{\text {secu }} \geq \max _{i=1: N}\left(\frac{M_{i}}{C_{i}} v_{\max }\right)+\max _{i=1: N}\left(l_{i}\right)+\max _{j=1: N}\left(L_{j}\right)
$$

where $M_{i}$ is the mass of sailboat $i$ and $C_{i}$ is the tangential friction of $S_{i}$, which can be measured as described in next paragraph. Note the shortest closed-path which can be performed by $N$ sailboats has a length of $N d_{\text {secu }}$.

\section{Measurement of $C_{i}$}

Approximation of $C_{i}$ in (4) can be measured in practice. Consider sailboat is moving at the instant $t=0$ such $v_{i}(0)=v_{\text {ini }}$ and $\theta_{i}=\psi_{t w}+\frac{\pi}{2}$. At instant $t=0^{+}$, open completely the sail such wind is not a driving force anymore, and measure the stopping distance $d_{\text {stop }, i}$ between $t=0^{+}$and instant $t=t_{f}$ where $v_{i}\left(t_{f}\right)=0$. One has $C_{i}=\frac{M_{i}}{d_{\text {stop }, i}} v(0)$. 


\subsection{Control of orientation and tack strategy}
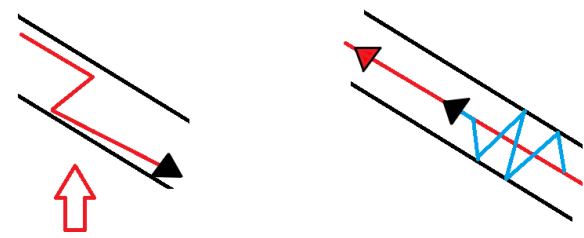

Fig. 3. Left: tack to move front the wind, represented by the red arrow. Right: tack to slow down and take distance between the two sailboats

Tuning vehicle acceleration is essential to perform a platooning. Theoretically, sailboat acceleration can be managed using the sail, using a method like sail control exposed in Section 4.2. However, maximal sailboat deceleration using the sail angle is very limited and not enough to perform platooning. Thus, to obtain an average acceleration in a particular direction, a solution is to use the rudder to make tacks inside a small corridor, like in Filippov's continuation method.

A control of the sailboat orientation $\theta_{i}$ to manage $\dot{\bar{v}}_{i}^{p r o j}$, while following platooning direction and stay inside a defined corridor, is proposed. Figures 2 and 3 show a corridor of width $2 r_{\max }$.

Let define $\bar{\theta}_{i}$ the desired orientation. If the acceleration of the boat at a time $t$ is not the desired acceleration $\dot{\bar{v}}^{\text {proj }}$, the sailboat makes tacks following orientation $\bar{\theta}_{i}=\beta_{i}+q_{i} \bar{\gamma}_{i}$, where $\bar{\gamma}_{i} \in\left[0, \frac{\pi}{2}\right]$ and $q_{i} \in\{-1,1\}$ such as $\dot{v}_{i}^{\text {proj }}=\dot{v}_{i} \cos \left(\bar{\theta}_{i}-\beta_{i}\right)=\dot{v_{i}} \cos \left(\bar{\gamma}_{i}\right)$. The value of $q_{i}$ allows to alternate the tack orientation when the sailboat reaches the limit of the corridor around line $\overline{\mathrm{O}}_{i} \underline{\mathrm{O}}_{i}$, as illustrated in Figure 3. $q_{i}$ is updated when $r>r_{\max }$. To avoid the sailboat turning back, we decide to limit $\dot{v}_{i}^{\text {proj }}$ to the domain $\left[0, \dot{v}_{i}\right]$, thus, $\bar{\gamma}_{i}$ is limited such as $\bar{\gamma}_{i} \in\left[0, \frac{\pi}{2}\right]$. By making tack, the boat goes at an average acceleration $\dot{\bar{v}}^{\text {proj }}$ as required in previous section.

The other problem is the wind orientation. To avoid the dead area where the boat is unable to move, tacking manoeuvres are needed. The tack directions are $\theta_{i}=$ $\psi+\pi+\delta$ and $\theta_{i}=\psi+\pi-\delta$, respectively. To reduce the risk of conflicting with the orientation $\bar{\theta}_{i}$ exposed in the previous paragraph if $\bar{\theta}_{i} \in\left[\psi_{t w}+\pi-\delta, \psi_{t w}+\pi+\delta\right]$ and not turn back, the closer hauled angle of the desired direction $\bar{\theta}_{i}$ and path orientation $\beta_{i}$ is selected. The discrete variable $q_{i}$ will automatically change tack when the sailboat is to leave the corridor.

The method to define the desired orientation $\bar{\theta}_{i}$ is described with the following steps:

- Step 0: First, to avoid a low velocity $v$, we check if $v_{i}$ is larger than a minim velocity $v_{\min }>0$. Else, using $k_{a}>0$, the desired acceleration is updated as

$$
\text { If } v_{i}<v_{\min }, \dot{\bar{v}}_{i}^{p r o j}=\max \left(\dot{\bar{v}}_{i}^{p r o j}, k_{a}\left(v_{\min }-v_{i}\right)\right)
$$

- Step 1: If $|r|>r_{\max }$, the sailboat is quite far from the line $\overline{\mathrm{O}}_{i} \underline{\mathrm{O}}_{i}$ : the tack variable $q_{i}$ is allowed to change its values such $q_{i}=\operatorname{sign}\left(\sin \left(\alpha_{i}\right)\right)$. Moreover, take $\bar{\gamma}_{i}=\frac{\pi}{2}-\varepsilon$ to move back into the corridor.

Else, if the acceleration $\dot{v}_{i}^{\text {proj }}$ of the sailboat is smaller or equal to the desired acceleration $\dot{\bar{v}}_{i}^{p r o j}$, i.e. if $\dot{v}_{i}^{\text {proj }} \leq$ $\dot{\bar{v}}_{i}^{\text {proj }}$, the most direct way to reach the objective is selected, i.e. $\bar{\gamma}_{i}=0$. Else, the rudder is used to regulate the deceleration. One has

$$
\begin{aligned}
& \text { If }|r|>r_{\max }, \text { then } q_{i}=\operatorname{sgn}\left(\sin \left(\alpha_{i}\right)\right) \\
& \text { Else } \\
& \bar{\gamma}_{i}=\frac{\pi}{2}-\varepsilon \\
& \bar{\gamma}_{i}=\min \left[\frac{\pi}{2}, \operatorname{acos}\left[\operatorname{sat}_{1}\left|\frac{\max \left(\dot{\bar{v}}_{i}^{\text {proj }}, 0\right)}{\max \left(\dot{v}_{i}, \varepsilon_{0}\right)}\right|\right]\right]
\end{aligned}
$$

A first evaluation of the desired orientation $\theta_{i}^{*}$ is computed using $\gamma_{i}$

$$
\theta_{i}^{*}=q_{i} \bar{\gamma}_{i}+\beta_{i}
$$

- Step 2: Check the wind orientation. If $\cos \left(\psi_{t w}-\theta_{i}^{*}\right)+\cos (\delta)>0$, compute $\bar{\theta}_{i}=\theta_{i}^{*}$. Else, $\theta_{i}^{*}$ is inside the dead area and need to be adjusted. However, the sailboat needs to continue to follow the path direction $\overline{\mathrm{O}}_{i} \underline{\mathrm{O}}_{i}$ and does not turn back. Moreover, we desire the sailboat to make the larger tack possible inside the corridor. Thus, a new orientation is defined close to the hauled angle:

If $\cos \left(\psi_{t w}-\theta_{i}^{*}\right)+\cos (\delta)>0, \bar{\theta}_{i}=\theta_{i}^{*}$

Else :

If $\cos \left(\beta_{i}-\left(\psi_{t w}+\pi-\delta\right)\right)<0, \bar{\theta}_{i}=\psi_{t w}+\pi+\delta$

Else if $\cos \left(\beta_{i}-\left(\psi_{t w}+\pi+\delta\right)\right)<0$,

$\bar{\theta}_{i}=\psi_{t w}+\pi-\delta$

Else $\bar{\theta}_{i}=\psi_{t w}+\pi+q_{i} \delta$

- Step 3: Evaluate $\delta_{s}$ using $\dot{v}_{i}^{p r o j}, \bar{\theta}_{i}$ as in Section 4.2.

Note this approach only requires the knowledge of sailboat parameters $M_{i}, l_{i}$ and $L_{i}$, making it simple to implement. Stability and convergence of the proposed method is given in Appendix A.1.

\subsection{Desired acceleration}

In this section, a platooning control is exposed. This one defines the desired acceleration on the axis $\overline{\mathrm{O}}_{i} \underline{\mathrm{O}}_{i}$ required to reach the platooning, used in Section 3.1 to define the desired orientation $\bar{\theta}_{i}$. Note this method can be exchanged with other ones in the literature, but due to the fact it is complex for a sailboat to respect a specific acceleration value, a more complex control does not guarantee a better platooning. 


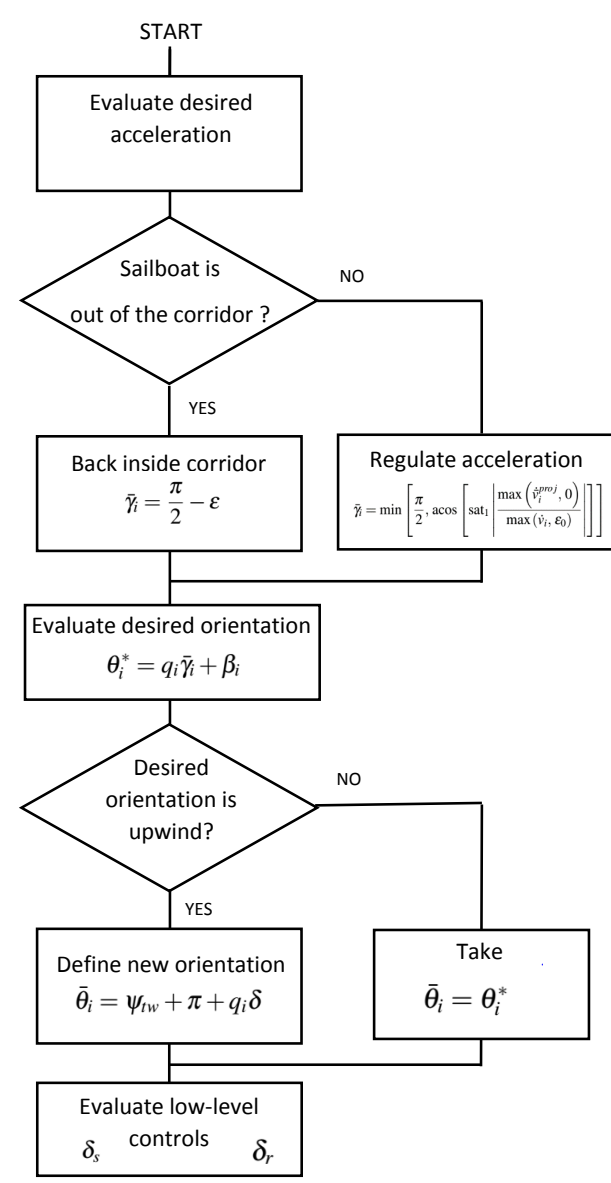

Fig. 4. Platooning control

We desire the sailboat projection velocity $v_{i}^{p r o j}$ converges to a targed value $\bar{v}_{0}$ and $d_{i, i-1}$ converges to the distance $d_{\text {secu }}=\frac{\|\mathrm{AB}\|+\|\mathrm{BC}\|+\|\mathrm{CD}\|+\|\mathrm{DA}\|}{N}$ if a closed-loop is considered, $d_{\text {secu }}>0$ a chosen value else. To obtain it, let define the desired acceleration $\dot{\bar{v}}_{i}^{p r o j}$ of the sailboat $i$ to reach the platooning.

To simplify the system analysis, the continuous-time dynamics of sailboat $i$ in a platooning is modelled by a second order equation:

$$
\begin{aligned}
M_{i} \dot{\bar{v}}_{i}^{\text {proj }} & =u_{i} \\
u_{i} & =-\sum_{j \in \mathcal{N}_{i}}\left[k_{p} \operatorname{sign}(j-i)\left(d_{i j}-|j-i| d_{\text {secu }}\right)\right. \\
& \left.+k_{v}\left(\bar{v}_{i}^{\text {proj }}-\bar{v}_{j}^{\text {proj }}\right)\right]-k_{0}\left(\bar{v}_{i}^{\text {proj }}-\bar{v}_{0}\right)
\end{aligned}
$$

where $k_{v}>2 \sqrt{k_{p} M_{i}}, k_{p}>0, k_{0} \geq 0$ if $k_{v}>0$ or $k_{0}>0$ if $k_{v} \geq 0, \bar{v}_{0}$ the desired platooning velocity. It is advised to take $k_{v}=2 \sqrt{k_{p} M_{i}}$ and $k_{p}=M_{i}$ to obtain an optimal convergence and stability.

Theorem 1: Consider a fleet of $N$ vehicles with dynamics given by (13)-(14). The fleet converges to a stable platooning such $\lim _{t \rightarrow \infty} \sum_{i=1}^{N} \sum_{j \in \mathcal{N}_{i}}\left\|d_{i j}(t)-|j-i| d_{\text {secu }}(t)\right\|^{2}=0 \quad$ and $\lim _{t \rightarrow \infty} \sum_{i=1}^{N}\left\|v_{i}-v_{0}\right\|^{2}=0$.

Proof of convergence and stability of Theorem 1 is provided in Appendix 1.

The desired acceleration to obtain the platooning is now obtained. However, the acceleration of a sailboat is limited by the wind velocity and it is difficult to control, thus this value may not always be respected.

\section{LOW LEVEL CONTROL}

In this section, low level controls of the sail and rudder angles are explored to follow the desired heading.

Proof of stability of the proposed method is shown in Appendix 1.

\subsection{Rudder angle}

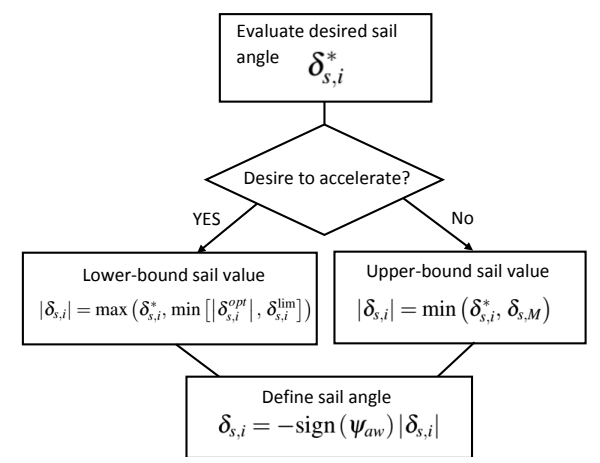

Fig. 5. Sail angle control

Remind that $\bar{\theta}_{i}$ is the desired orientation. In classic methods, if the boat has a consistent direction, a proportional control with respect to the error $\sin \left(\theta_{i}-\bar{\theta}_{i}\right)$ is performed. Else, if the sailboat orientation is far of $\bar{\theta}_{i}$, i.e. $\cos \left(\theta_{i}-\bar{\theta}_{i}\right)<0$, the rudder is tuned at its maximal orientation $\delta_{r, \text { max }}$. However, due to the sideways force of the wind, the course angle $\phi_{i}$ and the heading angle $\theta_{i}$ are not necessarily equal, as exposed in [9]. This effect is mainly observed when sailing close hauled, and $\phi_{i}$ and $\theta_{i}$ directions can be opposite when the boat is front to the wind. This discrepancy makes the sailboat diverge from the following line. Thus, a rudder control method of using course angle to compensate perturbations is proposed. Moreover, to avoid particular cases where $\theta_{i}$ and $\phi_{i}$ directions are opposite, the rudder control is described as follows

$$
\begin{aligned}
& \delta_{r}=\delta_{r, \max } \sin \left(\Theta_{i}-\bar{\theta}_{i}\right) \quad \text { if } \cos \left(\Theta_{i}-\bar{\theta}_{i}\right) \geq 0 \\
& \delta_{r}=\delta_{r, \max } \operatorname{sign}\left(\sin \left(\Theta_{i}-\bar{\theta}_{i}\right)\right) \quad \text { else. }
\end{aligned}
$$

where

$$
\begin{array}{ll}
\Theta_{i}=\phi_{i} & \text { if } \cos \left(\theta_{i}-\phi_{i}\right)-\cos \left(\varepsilon_{\theta}\right) \geq 0 \\
\Theta_{i}=\theta_{i} & \text { else. }
\end{array}
$$


with $\varepsilon_{\theta} \in\left[0, \frac{\pi}{2}[\right.$ a design parameter angle.

\subsection{Sail angle}

To help strategy development in Section 3.1 and obtain a smoother behavior, sail adjustment $\delta_{s, i}$ is managed to regulate the acceleration $\dot{v}_{i}$ of the boat. Due to the nonlinearity of the sailboat dynamics and as shown in [26], it is not possible to choose the velocity $v_{i}$ of the sailboat by linearizing outputs because of the singularities that can be incurred. Instead of that, the sail adjustment $\delta_{s, i}$ can be chosen to be as close as possible of the desired speed $\bar{v}_{i}$ or acceleration $\dot{\bar{v}}_{i}$. However, the main inconvenience of methods like in [26] is the knowledge of dynamic parameters of the sailboat, which is difficult to obtain. Thus, a control of the $\delta_{s, i}$ without using dynamic parameters and allowing to control the sailboat acceleration is proposed.

Note first since sail cannot hold against the wind, the angle of the sail $\delta_{s}$ cannot exceed a limit angle defined by the apparent wind. This conditions can be expressed like in [5] as

$$
\delta_{s} \in-\operatorname{sign}\left(\psi_{a w}\right) \min \left(|\pi-| \psi_{a w}||, \delta_{s, \max }\right)
$$

Let put the limit angle $\delta_{s, M}=\min \left(|\pi-| \psi_{a w}||, \delta_{s, \max }\right)$. Moreover, the optimal sail angle to move at maximal velocity is $\delta_{s, i}^{o p t}=\frac{\pi}{2}\left(\frac{\cos \left(\psi_{t w}-\bar{\theta}_{i}\right)+1}{2}\right)$ as exposed in [1].

Define the control of the sail with the following steps.

- Let define the angular acceleration such

$$
\dot{\delta}_{s, i}^{*}=-k_{s}\left(\dot{v}_{i}^{*}-\dot{v}_{i}\right) S_{\delta}
$$

with $k_{s}>0$ a design parameter, where $\breve{\delta}_{s}=\delta_{s}^{o p t}$ and $S_{\delta}=\left|\delta_{s}\right|-\left|\delta_{s}^{\text {opt }}\right|$ if $\dot{v}_{i}^{*}-\dot{v}_{i}>0, \breve{\delta}_{s}=\delta_{s, M}$ and $S_{\delta}=1$ else. Using a discrete step $d t$, the angle obtained using (20) is expressed as

$$
\delta_{s, i}^{*}(t)=\left|\delta_{s, i}(t)\right|+\dot{\delta}_{s, i}^{*} d t .
$$

Since we are working with a positive value of $\delta_{s, i}^{*}(t)$, if $\delta_{s, i}^{*}(t)<0$, take $\delta_{s, i}^{*}(t)=0$.

- If $\dot{\delta}_{s, i}^{*}<0$, we desire to speed up. Thus, one takes

$$
\left|\delta_{s, i}\right|=\max \left(\delta_{s, i}^{*}, \min \left[\left|\delta_{s, i}^{o p t}\right|, \delta_{s, i}^{\lim }\right]\right)
$$

where $\delta_{s, i}^{\lim }=\max \left(\delta_{s, M}-\varepsilon_{\delta_{s, i}}, 0\right)$. Term $\varepsilon_{\delta_{s, i}}$ is used to avoid sail and apparent wind are aligned, and so the sailboat can stay immobilized.

- If $\dot{\delta}_{s, i}^{*}>0$, we desire to slow down. Since the limit angle is defined by $\delta_{s, M}$, one takes

$$
\left|\delta_{s, i}\right|=\min \left(\delta_{s, i}^{*}, \delta_{s, M}\right)
$$

- Finally, one chooses $\delta_{s, i}=-\operatorname{sign}\left(\psi_{a w}\right)\left|\delta_{s, i}\right|$.

The main advantage of this technique is that the control of the sailboat acceleration can be obtained without knowledge of sailboat dynamic parameters. Moreover, this technique is simple to implement with a modest calculation time.

\section{SIMULATION}

\subsection{Model equations}

In this section, all sailboats are described by the following non-linear differential state equations developed in [5]

$$
\begin{aligned}
\dot{x}_{i} & =v_{i} \cos \left(\theta_{i}\right)+p_{1} a_{t w} \cos \left(\psi_{t w}\right) \\
\dot{y}_{i} & =v_{i} \sin \left(\theta_{i}\right)+p_{1} a_{t w} \sin \left(\psi_{t w}\right) \\
\dot{\theta}_{i} & =\omega_{i} \\
\dot{v}_{i} & =\left(g_{s} \sin \left(\delta_{s}\right)-g_{r} p_{11} \sin \left(\delta_{r}\right)-p_{2} v_{i}^{2}\right) / p_{9} \\
\dot{\omega}_{i} & =\left(g_{s}\left(p_{6}-p_{7} \cos \left(\delta_{s}\right)\right)-g_{r} p_{8} \cos \left(\delta_{r}\right)\right. \\
& \left.-p_{3} \omega v_{i}\right) / p_{10}
\end{aligned}
$$

where $g_{s, i}$ and $g_{r, i}$ are forces on sail and rudder, $W_{p, t w}=$ $\left[a_{t w}, \psi_{t w}\right]$ is the true wind defined in Section 2.1 and all parameters can be found in Table 1. $p_{2} v_{i}{ }^{2}$ and $p_{3} \omega_{i} v_{i}$ represent the tangential friction force and the angular friction force, respectively. The rudder force and the sail force are given by

$$
\begin{aligned}
& g_{r, i}=p_{5} v_{i}^{2} \sin \left(\delta_{r, i}\right) \\
& g_{s, i}=p_{4} a_{a w} \sin \left(\delta_{s, i}-\psi_{a w}\right)
\end{aligned}
$$

where $W_{p, a w}=\left[a_{a w}, \psi_{a w}\right]$ is the apparent wind.

\subsection{Simulation parameters}

The performance of the proposed method is evaluated considering a set of $N=8$ sailboats. Each sailboat $i$ can communicate with its immediate predecessor $i-1$ and follower $i+1$. The same graph is used to define platooning between sailboats. The four objectives are $\mathrm{A}=\left[\begin{array}{ll}500 & 0\end{array}\right], \mathrm{B}=\left[\begin{array}{ll}0 & 200\end{array}\right], \mathrm{C}=\left[\begin{array}{ll}-500 & 0\end{array}\right]$ and $D=\left[\begin{array}{ll}0 & -200\end{array}\right]$. Initial positions of sailboats are

$$
\begin{aligned}
& P 1=\left[\begin{array}{ll}
500 & 0
\end{array}\right] P 5=\left[\begin{array}{ll}
-500 & 0
\end{array}\right] \\
& P 2=\left[\begin{array}{ll}
235 & 100
\end{array}\right] P 6=\left[\begin{array}{ll}
-235 & -100
\end{array}\right] \\
& P 3=\left[\begin{array}{ll}
0 & 200
\end{array}\right] P 7=\left[\begin{array}{ll}
0 & -200
\end{array}\right] \\
& P 4=\left[\begin{array}{ll}
-265 & 100
\end{array}\right] P 8=\left[\begin{array}{ll}
265 & -100
\end{array}\right]
\end{aligned}
$$

Model parameters of sailboats are expressed in Table 1. The desired distance between sailboats is evaluated as $d_{\text {secu }}=\frac{\|\mathrm{AB}\|+\|\mathrm{BC}\|+\|\mathrm{CD}\|+\|\mathrm{DA}\|}{N}=269$. We choose $k_{p}=4$, $k_{v}=2, k_{0}=2, \varepsilon=\varepsilon_{\delta_{s}}=\frac{\pi}{36}$ and $\varepsilon_{\theta}=\frac{\pi}{8}$. The platooning target velocity is $v_{0}=2.5$ and minimum velocity $v_{\min }=1$. The wind orientation and strength are $\psi^{t w}=\frac{\pi}{2}$ and $a^{t w}=10$.

Two cases are compared in this section, respectively:

Case 1: the optimal receding horizon control (RHC) algorithm exposed in [21] for nonlinear vehicles. Algorithm is used to evaluate $u_{i}=\left[\boldsymbol{\delta}_{s, i}, \boldsymbol{\delta}_{r, i}\right]$ such sailboats converge to the platooning position and velocity. The dynamics model described by (24)-(28) is used. As method 


\begin{tabular}{|c|c|c||c|c|c|c|c|c|}
\hline & parameter & value & & parameter & value & & parameter & value \\
\hline$p_{1}$ & drift coefficient & 0.03 & $p_{5}\left[\mathrm{kgs}^{-1}\right]$ & rudder lift & 1500 & $p_{9}[\mathrm{~kg}]$ & mass of boat & 300 \\
\hline$p_{2}\left[\mathrm{kgs}^{-1}\right]$ & tangential friction & 40 & $p_{6}[\mathrm{~m}]$ & distance to sail & 0.5 & $p_{10}\left[\mathrm{kgm}^{2}\right]$ & moment of inertia & 400 \\
\hline$p_{3}[\mathrm{kgm}]$ & angular friction & 6000 & $p_{7}[\mathrm{~m}]$ & distance to mast & 0.5 & $p_{11}-$ & rudder break coefficient & 0.2 \\
\hline$p_{4}\left[\mathrm{kgs}^{-1}\right]$ & sail lift & 200 & $p_{8}[\mathrm{~m}]$ & distance to rudder & 2 & & & \\
\hline
\end{tabular}

Table 1. Model parameters value, from [5]

is developed for 1-dimensional space, projection of $P_{i}$, $v_{i}, \dot{v}_{i}$ on $\mathrm{ABCD}$ is considered to perform it. Constraints $u_{i} \in\left[\left[-\frac{\pi}{2}, \frac{\pi}{2}\right] \times\left[-\frac{\pi}{4}, \frac{\pi}{4}\right]\right]$ and $P_{i}$ inside the corridor are taken.

Case 2: heading control based on the regulated sail angle in Section 4.2 and tack strategy in Section 3.1 is used. Deceleration is guaranteed only by the sail angle and the tack strategy. $k_{s}=5$ and $d t=0.01$ are taken.

\subsection{Results}

Results of Case 1 are presented in Figure 6(a)(c) and results of Case 2 are presented in Figure 6(b)(d). In Case 2, velocity and distance between sailboats are maintained close to their desired value $v_{0}$ and $d_{\text {secu }}$. In Case 1, RHC algorithm allows to come close to $v_{0}$ and $d_{\text {secu }}$, but results are less stable and accurate than in Case 2. Problems of Case 1 come from the dependence of sailboat velocity with wind velocity/orientation: in some cases, RHC leads sailboat to configurations where velocity is difficult to increase (upwind) or decrease (back wind) for future manoeuvre. A larger prediction time would be required to avoid these problems. In Case 2, strategies like Step 4 (9)-(12) allow preventing these configurations, specific to sailboat constraints. Platooning is performed in both case, but we can note the method developed in Case 2 does not require knowledge of sailboat dynamics, which is difficult to obtain in practice, making it simpler to implement.

Video of the simulation can be found on https://www.youtube.com/watch?v=C72Fy6BskGY.

\section{CONCLUSION}

In this paper, a platooning method for sailboats has been defined, independent of sailboat dynamic models. A method to obtain the control of the boat acceleration for implementing platooning is proposed. To perform it, a tack strategy is described to regulate the projection of the sailboat acceleration in the main direction. This strategy is the main contribution of this paper because platooning cannot be implemented without it. A controller for rudder and angular sail are also proposed, and a desired acceleration of the sailboat is exposed. Proof of convergence of the platooning with the desired acceleration has been provided. Simulations show the effectiveness of the approach.

In future works, this method will be implemented on a fleet of autonomous sailboats for experimental validation. Moreover, the management of communications, like reducing the number of exchanges using the event-triggered approach, will be considered.

\section{ACKNOWLEDGMENT}

This work was supported by the Engineering and Physical Sciences Research Council (EPSRC) of the U.K. under Grant EP/R005532/1.

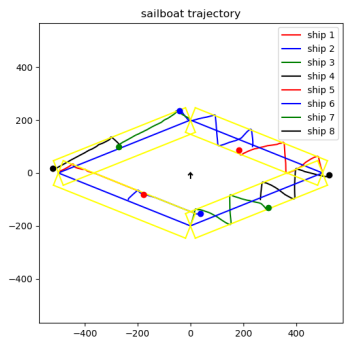

(a) Case 1: RHC algorithm
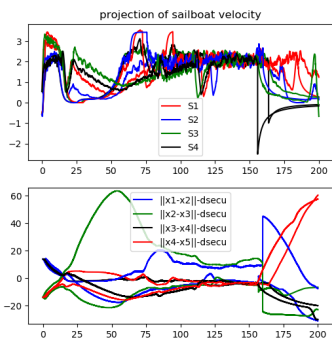

(c) Case 1: RHC algorithm

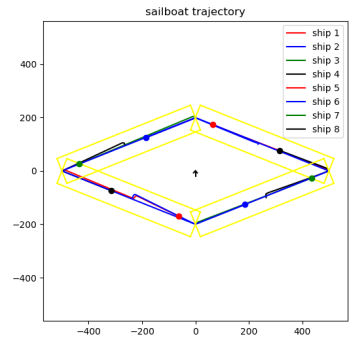

(b) Case 2: heading control
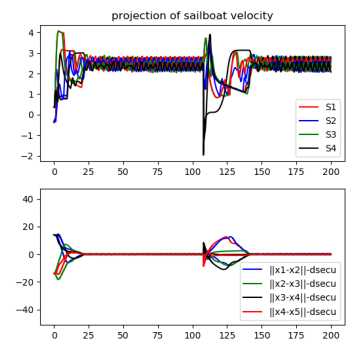

(d) Case 2: heading control
Fig. 6. Comparison of platooning methods. Distance are in $m$ and velocity in $m / s$

\section{APPENDIX A}

\section{PROOF OF PLATOONING CONVERGENCE}

To prove the convergence of the system and its stability, let's first rewrite (14) using the distance between agents and the sailboat $N$. Since $d_{N, i} \geq d_{N, j}$ if $i<j$, then 
$\operatorname{sign}(j-i) d_{i j}=\left(d_{N, i}-d_{N, j}\right)$ and one get

$$
\begin{aligned}
u_{i} & =-\sum_{j \in \mathcal{N}_{i}}\left[k_{p}\left(\left(d_{N, i}-d_{N, j}\right)-k_{p} \operatorname{sign}(j-i)|j-i| d_{\text {secu }}\right)\right. \\
& \left.+k_{v}\left(\bar{v}_{i}^{\text {proj }}-\bar{v}_{j}^{\text {proj }}\right)\right]-k_{0}\left(\bar{v}_{i}^{p r o j}-\bar{v}_{0}\right)
\end{aligned}
$$$$
=-\sum_{j \in \mathcal{N}_{i}}\left[k_{p}\left(\left(d_{N, i}-d_{N, j}\right)-(j-i) d_{\text {secu }}\right)\right.
$$$$
\left.+k_{v}\left(\dot{d}_{N, i}-\dot{d}_{N, j}\right)\right]-k_{0}\left(\dot{d}_{N, i}-\bar{v}_{0}^{*}\right)
$$

with $\bar{v}_{0}^{*}=\bar{v}_{0}-\bar{v}_{N}^{\text {proj }}$. Put $X=\left[\begin{array}{llll}d_{N, 1} & d_{N, 2} & \ldots & d_{N, N}\end{array}\right]^{T}$, $M=\operatorname{diag}\left(\left[M_{1}, \ldots, M_{N}\right]\right)$ and $V=\dot{X}$. Using (A.1), the system (13)-(14) can be rewritten as

$$
\begin{aligned}
M \dot{V} & =U \\
U & =-k_{0}\left(V-V_{0}\right)-k_{p}(L X+D)-k_{v} L V
\end{aligned}
$$

with $L$ the Laplacian matrix of the graph $\mathcal{G}, \quad V=\left[\begin{array}{llll}\bar{v}_{1}^{\text {proj }} & \bar{v}_{2}^{\text {proj }} & \ldots & \bar{v}_{N}^{\text {proj }}\end{array}\right]^{T}, \quad U=$ $\left[\begin{array}{llll}u_{1} & u_{2} & \ldots & u_{N}\end{array}\right], V_{0}=1_{N} \bar{v}_{0}$ and $D=\left[d_{i}\right]_{i=1 \ldots N}$ with $d_{i}=\sum_{j=1}^{N} a_{i j}(i-j) d_{\text {secu }}$.Put $V^{*}=V-V_{0}$ and observe $L V_{0}=L 1_{N} v_{0}=0_{N}$ so $L V^{*}=L V$ and $\dot{V}^{*}=\dot{V}$. Then, (A.2) can be expressed as

$$
U=-k_{0} V^{*}-k_{p}(L X+D)-k_{v} L V^{*}
$$

Consider the following candidate Lyapunov function $\mathbb{V}$

$$
\mathbb{V}=\frac{1}{2}(L X+D)^{T}(L X+D)+\frac{1}{2} V^{* T} V^{*}=\frac{1}{2} W^{T} W
$$

with $W=\left[\begin{array}{cc}V^{*} & L X+D\end{array}\right]^{T} . \mathbb{V}$ can be expressed as

$$
\begin{aligned}
{\left[\begin{array}{c}
\dot{V}^{*} \\
L V^{*}
\end{array}\right] } & =\left[\begin{array}{cc}
-k_{0} M^{-1}-k_{v} M^{-1} L & -k_{p} M^{-1} \\
L & 0_{N}
\end{array}\right]\left[\begin{array}{c}
V^{*} \\
L X+D
\end{array}\right] \\
\dot{W} & =A W
\end{aligned}
$$

and since $L$ is definite positive, $k_{p}>0$, and $k_{v}>2 \sqrt{k_{p} M_{i}}$ or $k_{0}>0$, it can be shown $A$ is definite negative.

Taking the derivative of $\mathbb{V}$, one has

$$
\dot{\mathbb{V}}=W^{T} \dot{W}=W^{T} A W \leq 0
$$

According to the hypotheses of the Lyapunov theory, the system is stable.

$$
\dot{\mathbb{V}} \leq-\alpha W^{T} W=-\alpha \mathbb{V}
$$

where $\alpha=\left|\lambda_{\max }(A)\right|$. Let define the function $\mathbb{Z}$ such $\dot{\mathbb{Z}}(t)=-\alpha \mathbb{Z}(t)$ and $\mathbb{Z}(0)=\mathbb{V}(0)$. We can easily show than $\mathbb{Z}(t)=\mathbb{V}(0) e^{-\alpha t}$. Using the comparison theorem [27], one has $\mathbb{V}(t) \leq \mathbb{Z}(t)$, so $\mathbb{V}(t) \leq \mathbb{V}(0) e^{-\alpha t}$, so $\mathbb{V}(t)$ converges to zero when $t$ converges to $\infty$. Thus $\|L X+D\|$ and $V^{*}$ converge to zero, and so $\bar{v}_{i}^{p r o j}$ converges to $\bar{v}_{0}$ for all $i \in \mathcal{N}$ and $d_{j, i}$ converges to $d_{\text {secu }}$.

\section{A.1. Proof of stability and convergence of control ori- entation}

Following steps described in Section 3.1, one get $\bar{\theta}_{i}=$ $q_{i} \bar{\gamma}_{i}+\beta_{i}$. As shown in Appendix B.3, control of the rudder allows a stable convergence from $\theta_{i}$ to $\bar{\theta}_{i}$. Since $\dot{v}_{i}^{p r o j}=\dot{v}_{i} \cos \left(\bar{\theta}_{i j}-\beta_{i}\right)=\dot{v}_{i} \cos \left(q_{i} \bar{\gamma}_{i}\right)$, and since (7), one has $\dot{v}_{i}^{\text {proj }}=\dot{\bar{v}}_{i}^{\text {proj }}$ if $\theta_{i}$ has converged to $\bar{\theta}$. To conclude, since $\dot{\bar{v}}_{i}^{\text {proj }}$ guarantees a stable convergence to the platooning and rudder guarantees a stable convergence from $\dot{v}_{i}^{p r o j}$ to $\overline{\bar{v}}_{i}^{p r o j}$, control orientation is stable and platooning tracking error converges to zero.

\section{APPENDIX B}

\section{STABILITY AND CONVERGENCE OF RUDDER CONTROL}

The stability of a rudder and a sail control for a general nonlinear dynamic model of a sailboat is shown.

\section{B.1. Sailboat dynamics model}

As shown in [9], a general nonlinear dynamic of a sailboat rotation can be expressed as

$$
J \ddot{\phi}=\tau_{r}+\tau_{s}+\tau_{k}+\tau_{h}
$$

where $J$ is sailboat inertia matrix, $\phi$ course angle of the sailboat, $\tau_{r}, \tau_{s}, \tau_{k}, \tau_{h}$ are the rudder, sail, keel and hydrodynamics actions. In particular, one may write $\tau_{r} \approx$ $-\beta v^{2} \sin \left(2 \delta_{r}\right)$ where $\delta_{r}$ is the rudder angle, $v$ the sailboat velocity, and $\beta>0$ a constant based on sailboat shape, water density, lift and drag coefficients. Knowledge of $J, \tau_{s}, \tau_{k}, \tau_{h}$ and $\beta$ are not required to implement the following proof.

\section{B.2. Proof of stability}

Let define $\omega=\dot{\phi}$. The desired rotation velocity $\bar{\omega}$ and desired rotation acceleration $\dot{\bar{\omega}}$ are chosen such that $\bar{\omega}=\omega-\sin (\Theta-\bar{\theta})$ and $\dot{\bar{\omega}}=0$. Thus, one can deduce

$$
\omega-\bar{\omega}=\sin (\Theta-\bar{\theta})
$$

Note $\dot{\bar{\omega}}$ tries to make $\dot{\omega}$ to converge to zero and $\bar{\omega}$ tries to make $\omega$ to converge to zero when the sailboat reached the desired orientation.

Define now the candidate Lyapunov function $V$ such

$$
V=\frac{1}{2} J(\omega-\bar{\omega})^{2}\left(1-\frac{1}{1+\ln (2-\cos (\Theta-\bar{\theta}))}\right)
$$

and put $W=1-\frac{1}{1+\ln (2-\cos (\Theta-\bar{\theta}))}$ such $W \geq 0$ and $W=0$ when $\Theta-\bar{\theta}=0$. The derivative of $V$ is

$$
\dot{V}=(\omega-\bar{\omega}) J(\dot{\omega}-\dot{\bar{\omega}}) W+\frac{1}{2} J(\omega-\bar{\omega})^{2} d W
$$


with

$$
\begin{aligned}
d W & =-\frac{-\left(\frac{\sin (\Theta-\bar{\theta})(\omega-\bar{\omega})}{2-\cos (\Theta-\bar{\theta})}\right)}{(1+\ln (2-\cos (\Theta-\bar{\theta})))^{2}} \\
& =-\frac{\sin (\Theta-\bar{\theta})(\omega-\bar{\omega})}{(2-\cos (\Theta-\bar{\theta}))(1+\ln (2-\cos (\Theta-\bar{\theta})))^{2}}
\end{aligned}
$$

Using (B.2), one has

$$
\begin{aligned}
d W & =-\frac{\sin (\Theta-\bar{\theta})^{2}}{(2-\cos (\Theta-\bar{\theta}))(1+\ln (2-\cos (\Theta-\bar{\theta})))^{2}} \\
& \leq 0
\end{aligned}
$$

Thus

$$
\dot{V} \leq(\omega-\bar{\omega}) J \ddot{\phi} W
$$

Using (B.1), (B.2) and put $S_{\tau}=\tau_{s}+\tau_{k}+\tau_{h}$, one gets

$$
\dot{V}=\sin (\Theta-\bar{\theta})\left(\tau_{r}+S_{\tau}\right) W
$$

Since the sailor can only use the rudder to control sailboat orientation and rudder action is limited by $\left|\tau_{r}\right| \leq$ $\beta v^{2} \sin \left(\delta_{r, \max }\right)$. If $\left|\tau_{r}\right| \leq\left|S_{\tau}\right|$ with $\operatorname{sgn}\left(\tau_{r}\right) \neq \operatorname{sgn}\left(S_{\tau}\right)$, then the sailboat orientation is uncontrollable. Then, suppose here we are in a case where sailboat is controllable, so $\operatorname{sgn}\left(\tau_{r}\right)=\operatorname{sgn}\left(S_{\tau}\right)$ or $\left|\tau_{r}\right|>\left|S_{\tau}\right|$. Thus, one may write $\tau_{r}+S_{\tau}=\alpha \operatorname{sgn}\left(\tau_{r}\right)$ where $\alpha>0$ (case $\alpha=0$ induce $\tau_{r}+S_{\tau}=0$, so sailboat is uncontrollable too. Note if $\tau_{r}+S_{\tau}=0$, one has $\left.\dot{V}=\frac{1}{2} J(\omega-\bar{\omega})^{2} d W \leq 0\right)$. Define also $\alpha_{\min }$ the smallest value of $\alpha \operatorname{such} \tau_{r}+S_{\tau}=\alpha \operatorname{sgn}\left(\tau_{r}\right)$ with $\operatorname{sgn}\left(\tau_{r}\right) \neq \operatorname{sgn}\left(S_{\tau}\right)$, i.e. $\alpha_{\min }=\min (\alpha) \forall\left(\tau_{r}, S_{\tau}\right) \neq$ $\{0,0\}$ and $\operatorname{sgn}\left(\tau_{r}\right) \neq \operatorname{sgn}\left(S_{\tau}\right)$. One gets

$$
\begin{aligned}
\dot{V} & \leq \sin (\Theta-\bar{\theta}) \alpha \operatorname{sgn}\left(\tau_{r}\right) W \\
& \leq \sin (\Theta-\bar{\theta}) \alpha \operatorname{sgn}\left(-\beta v^{2} \sin \left(2 \delta_{r}\right)\right) W \\
& \leq-\alpha \sin (\Theta-\bar{\theta}) \operatorname{sgn}\left(\sin \left(\delta_{r}\right) \cos \left(\delta_{r}\right)\right) W
\end{aligned}
$$

Remind $\delta_{r} \in\left[-\delta_{r, \text { max }}, \delta_{r \text {,max }}\right]$ where $0<\delta_{r, \text { max }}<\frac{\pi}{2}$, thus $\cos \left(\delta_{r}\right) \geq 0$. Put $S=|\sin (\Theta-\bar{\theta})|$ if $\cos (\Theta-\bar{\theta}) \geq 0$ and $S=1$ else. Using (15)-(16), one gets

$$
\begin{aligned}
\dot{V} & \leq-\alpha \sin (\Theta-\bar{\theta}) \operatorname{sgn}\left(\sin \left(\delta_{r, \max } S \operatorname{sgn}(\sin (\Theta-\bar{\theta}))\right)\right) W \\
& \leq-\alpha \sin (\Theta-\bar{\theta}) \operatorname{sgn}(\sin (\Theta-\bar{\theta})) W \\
& \leq-\alpha|\sin (\Theta-\bar{\theta})| W \leq 0
\end{aligned}
$$

Thus, $V \geq 0$ and $\dot{V} \leq 0$. Using the Lyapunov theorem, one deduces the rudder control (15)-(16) allows a stable control in orientation and rotation velocity if sailboat orientation is controllable by the rudder.

\section{B.3. Proof of convergence}

Let now show the convergence of $V$. Using (B.3), one has

$$
\dot{V} \leq-|\sin (\Theta-\bar{\theta})|\left(\frac{2 \alpha}{J}\right)\left(\frac{1}{2} J W \frac{(\omega-\bar{\omega})^{2}}{(\omega-\bar{\omega})^{2}}\right)
$$

and since $\omega-\bar{\omega}=\sin (\Theta-\bar{\theta})$, one has

$$
\begin{aligned}
\dot{V} & \leq-|\sin (\Theta-\bar{\theta})|\left(\frac{2 \alpha}{J}\right)\left(\frac{1}{2} J W \frac{(\omega-\bar{\omega})^{2}}{\sin (\Theta-\bar{\theta})^{2}}\right) \\
& \leq-\left(\frac{2 \alpha}{J}\right)\left(\frac{1}{2} J W \frac{(\omega-\bar{\omega})^{2}}{|\sin (\Theta-\bar{\theta})|}\right) \\
& \leq-\left(\frac{2 \alpha_{\min }}{J \omega_{\max }}\right) V
\end{aligned}
$$

Let define the function $U$ such $\dot{U}=-\left(\frac{4 \alpha_{\min }}{J \omega_{\max }}\right) U$ and $U(0)=V(0)$. We can easily show than $U(t)=$ $V(0) e^{-\left(\frac{4 \alpha_{\min }}{J \omega_{\max }}\right) t}$. Using the comparison theorem in [27], one has $V(t) \leq U(t)$, so

$$
V(t) \leq V(0) e^{-\left(\frac{4 \alpha_{\min }}{J \omega_{\max }}\right) t}
$$

From (B.4), one may deduce $\lim _{t \rightarrow \infty} V(t)=$ $\lim _{t \rightarrow \infty} V(0) e^{-\left(\frac{4 \alpha_{\min }}{J \omega_{\max }}\right) t}=0$. So, $V(t)$ converges to zero. Since $V=\frac{1}{2} J(\omega-\bar{\omega})^{2}\left(1-\frac{1}{1+\ln (2-\cos (\Theta-\bar{\theta}))}\right), V(t)=0$ induce $(\omega-\bar{\omega})=0$ or $\left(1-\frac{1}{1+\ln (2-\cos (\Theta-\bar{\theta}))}\right)=0$. Let study the two solutions.

$$
\text { If } \begin{aligned}
\left(1-\frac{1}{1+\ln (2-\cos (\Theta-\bar{\theta}))}\right)=0: & \\
1-\frac{1}{1+\ln (2-\cos (\Theta-\bar{\theta}))} & =0 \\
2-\cos (\Theta-\bar{\theta}) & =1 \\
\Theta & =\bar{\theta}
\end{aligned}
$$

If $(\omega-\bar{\omega})=0$, one has $(\omega-\bar{\omega})=0 \Leftrightarrow \sin (\Theta-\bar{\theta})=0$ $\Leftrightarrow \Theta=\bar{\theta}$. In the both case, one has $\Theta=\bar{\theta}$. One deduces the rudder control (15)-(16) allows $\Theta$ converging to the desired value $\bar{\theta}$.

\section{STABILITY OF SAIL CONTROL}

To prove the sail control stability exposed in Section 4.2., let define the Lyapunov function $V=$ $\frac{1}{2}\left\|\delta_{s}-\breve{\delta}_{s}\right\|^{2}$. Since the sail opening is controlled by the length of a rope, which is further controlled by a motor with a PID, we can assume than $\dot{\delta}_{s}=\dot{\delta}_{s}^{*} \operatorname{sign}\left(\delta_{s}\right)$. Thus, one has

$$
\dot{V}=\dot{\delta}_{s}\left(\delta_{s}-\breve{\delta}_{s}\right)
$$

Since $\operatorname{sign}\left(\delta_{s}\right)=\operatorname{sign}\left(\breve{\delta}_{s}\right)$ (wind orientation), and using (20), one gets

$$
\dot{V}=-k_{s}\left(\dot{v}^{*}-\dot{v}\right) S_{\delta}\left(\left|\delta_{s}\right|-\left|\breve{\delta}_{s}\right|\right)
$$


Consider now the two following case. First, if $\left(\dot{v}^{*}-\dot{v}\right) \leq$ 0, one has $\breve{\delta}_{s}=\delta_{s, M}$ and $S_{\delta}=1$, so $\left|\delta_{s}\right| \leq\left|\breve{\delta}_{s}\right|$ due to (19). Thus, one has $\dot{V} \leq 0$. Second, if $\left(\dot{v}^{*}-\dot{v}\right) \geq 0$, one has $\breve{\delta}_{s}=\delta_{s}^{o p t}$ and $S_{\delta}=\left|\delta_{s}\right|-\left|\delta_{s}^{o p t}\right|$, so

$$
\dot{V}=-k_{s}\left(\dot{v}^{*}-\dot{v}\right)|| \delta_{s}|-| \breve{\delta}_{s}|| \leq 0
$$

$\dot{V} \leq 0$ in all cases. Using the Lyapunov theorem, the sail control exposed in Section 4.2 is stable.

\section{REFERENCES}

[1] C. Viel, U. Vautier, J. Wan, L. Jaulin, Position keeping control of an autonomous sailboat, IFAC CAMS 51 (29),1419, 2018.

[2] J. Chen, Z. Ye, R. Yang, G. Cai, J. Li, H. Li, Design and control of multiple wing-sail land yacht robot, in: In Proc IEEE ICMA, pp. 1800-1805, 2018.

[3] T. Enqvist, A. Friebe, F. Haug, Free rotating wingsail arrangement for åland sailing robots, in: World Robotic Sailing championship and International Robotic Sailing Conference, pp. 3-18, 2016.

[4] F. Le Bars, L. Jaulin, An experimental validation of a robust controller with the vaimos autonomous sailboat, in: Robotic Sailing 2012, pp. 73-84, 2013.

[5] J. Melin, Modeling, control and state-estimation for an autonomous sailboat, 2015.

[6] F. Plumet, H. Saoud, M.-D. Hua, Line following for an autonomous sailboat using potential fields method, in: Proc MTS/IEEE, OCEANS-Bergen, pp. 1-6, 2013.

[7] M. Kang, J. Xu, J. Xu, M. Du, Autonomous sailboat local route planning, in: World Robotic Sailing championship and International Robotic Sailing Conference, pp. 33-43, 2016.

[8] J. Melin, K. Dahl, M. Waller, Modeling and control for an autonomous sailboat: a case study, in: Robotic Sailing 2015, pp. 137-149, 2016.

[9] H. Saoud, M.-D. Hua, F. Plumet, F. B. Amar, Routing and course control of an autonomous sailboat, in: Proc IEEE ECMR, pp. 1-6, 2015.

[10] D. Santos, A. G. Silva Junior, A. Negreiros, J. Vilas Boas, J. Alvarez, A. Araujo, R. V. Aroca, L. M. Gonçalves, Design and implementation of a control system for a sailboat robot, Robotics 5 (1), 2016.

[11] H. Chehardoli, A. Ghasemi, Adaptive centralized/decentralized control and identification of 1-d heterogeneous vehicular platoons based on constant time headway policy, In Proc IEEE Transactions on Intelligent Transportation Systems 1 (1), 1-11, 2018.

[12] M. Bernardo, P. Falcone, A. Salvi, S. Santini, Design, analysis, and experimental validation of a distributed protocol for platooning in the presence of time-varying heterogeneous delays, In Proc IEEE Transactions on Control Systems Technology 24 (2), 413-427, 2016.
[13] Y. A. Harfouch, S. Yuan, S. Baldi, An adaptive switched control approach to heterogeneous platooning with intervehicle communication losses, In Proc. IEEE Transactions on Control of Network Systems 5 (3), 1434-1444, 2018.

[14] S. Sabău, C. Oară, S. Warnick, A. Jadbabaie, Optimal distributed control for platooning via sparse coprime factorizations, In Proc IEEE Transactions on Automatic Control 62 (1), 305-320, 2017.

[15] A. Medina, N. Van de Wouw, H. Nijmeijer, Cooperative intersection control based on virtual platooning, In Proc IEEE Transactions on Intelligent Transportation Systems 19 (6), 1727-1740, 2018.

[16] B. Liu, D. Jia, K. Lu, D. Ngoduy, J. Wang, L. Wu, A joint control-communication design for reliable vehicle platooning in hybrid traffic, In Proc IEEE Transactions on Vehicular Technology 66 (10), 9394-9409, 2017.

[17] B. Béchadergue, L. Chassagne, H. Guan, Vehicle-tovehicle visible light phase-shift rangefinder based on the automotive lighting, In Proc IEEE Sensors Journal 18 (13), 5334-5342, 2018.

[18] V. Dolk, J. Ploeg, H. Heemels, Event-triggered control for string-stable vehicle platooning, In Proc. IEEE Transactions on Intelligent Transportation Systems 18 (12), 34863500, 2017.

[19] S. Linsenmayer, D. Dimarogonas, Event-triggered control for vehicle platooning, in: Proc IEEE ACC, pp. 31013106, 2015.

[20] C. Viel, S. Bertrand, M. Kieffer, H. Piet-Lahanier, Distributed event-triggered control for multi-agent formation stabilization, In Proc IFAC WC 50 (1), 8025-8030, 2017.

[21] H. Li, Y. Shi, W. Yan, Distributed receding horizon control of constrained nonlinear vehicle formations with guaranteed $\gamma$-gain stability, Automatica 68, 148-154, 2016.

[22] H. Li, P. Xie, W. Yan, Receding horizon formation tracking control of constrained underactuated autonomous underwater vehicles, In Proc. IEEE Transactions on Industrial Electronics 64 (6), 5004-5013, 2017.

[23] Z. Peng, J. Wang, D. Wang, Distributed containment maneuvering of multiple marine vessels via neurodynamicsbased output feedback, IEEE Transactions on Industrial Electronics 64 (5), 3831-3839, 2017.

[24] Z. Peng, J. Wang, Output-feedback path-following control of autonomous underwater vehicles based on an extended state observer and projection neural networks, In Proc. IEEE Trans. on Syst., Man, and Cyber.: Systems 48 (4), 535-544, 2018.

[25] Z. Peng, J. Wang, D. Wang, Distributed maneuvering of autonomous surface vehicles based on neurodynamic optimization and fuzzy approximation, In Proc IEEE Trans. on Control Syst. Techn. 26 (3), 1083-1090, 2018.

[26] P. Herrero, L. Jaulin, J. Vehí, M. Sainz, Guaranteed setpoint computation with application to the control of a sailboat, IJCAS 8 (1), 1-7, 2010.

[27] Nonlinear Systems, 3rd ed., Upper Saddle River,NJ: Prentice-Hall, 2002. 